\title{
Real-World Observational Evaluation of Hair Thinning in Patients with Multiple Sclerosis Receiving Teriflunomide: Is It an Issue in Clinical Practice?
}

\author{
Lori Hendin Travis · Annette Okai · Steve Cavalier · Darren Stam • \\ Lisa Farnett · Keith R. Edwards
}

Received: May 8, 2018 / Published online: August 6, 2018

(C) The Author(s) 2018

\begin{abstract}
Introduction: Hair thinning occurred in $10-14 \%$ of teriflunomide-treated patients in the teriflunomide multiple sclerosis clinical development program, compared with $5 \%$ of placebo-treated patients. Our objective was to examine the clinical course of hair thinning in patients in an observational real-world project. Methods: Patients with relapsing-remitting multiple sclerosis who reported hair thinning to healthcare professionals (HCPs) during treatment with teriflunomide were eligible for inclusion. During two office visits, one at onset of hair thinning and another at follow-up, HCPs and patients completed questionnaires that
\end{abstract}

Enhanced digital content To view enhanced digital content for this article go to https://doi.org/10.6084/ m9.figshare.6866177.

L. Hendin Travis ( $\square)$

Phoenix Neurological Associates Ltd, Phoenix, AZ, USA

e-mail: LHT@pnal.net

A. Okai

Multiple Sclerosis Treatment Center of Dallas,

Dallas, TX, USA

S. Cavalier - D. Stam - L. Farnett

Sanofi, Cambridge, MA, USA

K. R. Edwards

Multiple Sclerosis Center of Northeastern New York, Latham, NY, USA categorized hair thinning as mild, moderate, or severe, or from 0 (no hair thinning) to 10 (very severe hair thinning), respectively. At the follow-up visit, patients also rated the degree of recovery. Patients were photographed at both visits with a standardized protocol and camera. Results: Of the 38 patients who completed follow-up, most were women $(97 \%)$ without prior history of hair thinning (87\%), with the majority (68\%) receiving concomitant medications potentially associated with hair thinning. The mean time to onset of hair thinning was 77 days after the first teriflunomide dose. HCPs classified the majority of hair thinning events as mild $(63 \%)$ or moderate $(34 \%)$, with one event classified as severe (3\%). The mean patient severity perception was $5 / 10$, and complete/ near-complete resolution or marked improvement was reported by $79 \%$ of patients.

Conclusion: Consistent with observations from the teriflunomide clinical program, hair thinning was usually mild and occurred within the first 3 months of treatment, with most patients fully recovering while remaining on teriflunomide treatment. As with any potential adverse event, it is important to ensure appropriate expectations through patient education before treatment.

Funding: Sanofi.

Keywords: Multiple sclerosis; Real-world data; Safety; Teriflunomide 


\section{INTRODUCTION}

A number of different factors can contribute to hair loss, a distressing condition that negatively impacts patients' psychological and social wellbeing $[1,2]$. Hair loss can be triggered by emotional stress, diet, illness, underlying medical conditions, or exposure to medication; severe hair loss is often associated with chemotherapy or radiation treatments [3]. There have been reports of hair thinning in patients with multiple sclerosis (MS) treated with disease-modifying therapies (DMTs) [4-8].

Teriflunomide (Aubagio ${ }^{\circledR}$; Genzyme, Cambridge, MA) is a once-daily oral immunomodulator for the treatment of patients with relapsing-remitting MS (RRMS). In most countries, the approved dose of teriflunomide is $14 \mathrm{mg} /$ day; in the United States, the 7-mg daily dose is also approved. In a pooled analysis of clinical trials, hair loss was observed in 10-14\% of patients receiving teriflunomide $7 \mathrm{mg}$ or $14 \mathrm{mg}$, compared with $5 \%$ of those receiving placebo [9]. Most of these hair thinning events occurred within the first 6 months of treatment, were characterized by the investigators as mild or moderate in nature, and resolved without corrective treatment while patients remained on teriflunomide. Few patients $(<2 \%)$ who experienced hair thinning discontinued teriflunomide treatment for this reason, and no complete hair loss was reported [9].

The assessment and characterization of teriflunomide-associated hair thinning in realworld clinical practice settings may help to set appropriate expectations for patients before initiating treatment with teriflunomide. Here, we report an endeavor to acquire a better understanding of the clinical course of hair thinning in patients with MS treated with teriflunomide.

\section{METHODS}

\section{Study Population and Design}

This was an observational real-world evaluation conducted at nine MS centers in the United States between May 2013 and the data cutoff of
May 14, 2015. Patients with RRMS who reported hair thinning to healthcare professionals (HCPs) during treatment with teriflunomide $7 \mathrm{mg}$ or $14 \mathrm{mg}$ were eligible for inclusion.

\section{Patient Assessments}

During two office visits, one at the onset of hair thinning and another at follow-up, HCPs completed questionnaires with their patients. The description of hair thinning was reported. HCPs categorized hair thinning as mild, moderate, or severe, while patients ranked the severity of the event from 0 to 10. At the follow-up visit, HCPs and patients also rated the degree of improvement or resolution of hair thinning according to the categories listed in Table 1.

At onset and follow-up visits, patients were photographed from five standard views (anterior, posterior, left lateral, right lateral, anterior superior) and an optional manipulated view with hair pulled back, using a standardized protocol and camera.

\section{Compliance with Ethics Guidelines}

Informed consent was obtained from all individual participants included in the study. Additional written informed consent was obtained from participants for release of their images.

This trial was not registered on ClinicalTrials.gov because it is a real-world observational evaluation and not a clinical trial.

\section{RESULTS}

Of the 38 patients who completed follow-up visits at data cutoff, 36 were treated with teriflunomide $14 \mathrm{mg}$ and two were treated with teriflunomide $7 \mathrm{mg}$. The majority of patients were women $(97 \%)$, and the mean (standard deviation) age was 52 (12) years. Most had no prior history of hair loss (87\%), and many (68\%) were receiving concomitant medications associated with hair thinning, for conditions such as migraine, epilepsy, or depression [10]. 
Table 1 Healthcare professional (HCP) and patient rating of hair thinning

\begin{tabular}{ll}
\hline HCP-perceived severity at onset and follow-up visit & Mild \\
& Moderate \\
& Severe \\
Patient-perceived severity at onset visit & 0 (no hair thinning) to 10 (very severe hair thinning) \\
Patient-rated degree of improvement at follow-up visit & None/minimal \\
& Somewhat improved \\
& Markedly improved \\
& Complete/near-complete resolution \\
\hline
\end{tabular}

The mean time from the initiation of teriflunomide treatment to onset of hair thinning was 77 days ( $<3$ months), and the mean time from onset to follow-up visit was 270 days. At onset, the mean score for patient perception of severity was 5.0 (range 2-10; Table 2). HCPs classified the vast majority of cases as mild $(63 \%)$ or moderate $(34 \%)$, with a single severe case in a 33-year-old woman with a prior history of drug-induced hair loss with topiramate. Most patients (79\%) reported complete/near-complete resolution or marked improvement at follow-up. HCPs also reported lower severity in follow-up visits (Table 2). A single patient reported only minimal improvement, although the HCP rated that patient's hair loss as only mild at both visits. Overall, patient perception of hair thinning was more severe than HCP perception of hair thinning.

Photographic examples of hair thinning for eight patients at onset and follow-up are shown in Fig. 1. Patient and HCP assessments at each visit are provided.

Table 2 Patient and HCP perceptions of hair thinning events at onset and follow-up

\begin{tabular}{lll}
\hline Assessment & Onset visit $(\boldsymbol{N}=\mathbf{3 8})$ & Follow-up visit $(\boldsymbol{N}=\mathbf{3 8})$ \\
\hline Patient perception of severity, mean (range) & $5.0(2-10)$ & $3.5(1-9)$ \\
Patient rating of resolution, $n$ (\%) & & \\
Complete/near complete & - & $14(37)$ \\
Markedly improved & - & $16(42)$ \\
Somewhat improved & - & $7(18)$ \\
None/minimal & - & $1(3)$ \\
HCP perception of severity, $n(\%)$ & & \\
Mild & $24(63)$ & $31(82)$ \\
Moderate & $13(34)$ & $7(18)$ \\
Severe & $1(3)$ & 0 \\
\hline
\end{tabular}

$H C P$ healthcare professional 


\begin{tabular}{|c|c|c|c|c|c|c|c|c|}
\hline & \multicolumn{2}{|c|}{ Patient 1} & \multicolumn{2}{|c|}{ Patient 2} & \multicolumn{2}{|c|}{ Patient 3} & \multicolumn{2}{|c|}{ Patient 4} \\
\hline & Onset & Follow-up & Onset & Follow-up & Onset & Follow-up & Onset & Follow-up \\
\hline Age, years & \multicolumn{2}{|r|}{57} & \multicolumn{2}{|r|}{38} & \multicolumn{2}{|c|}{70} & \multicolumn{2}{|r|}{44} \\
\hline $\begin{array}{l}\text { Time from first dose of teriflunomide } \\
\text { to onset of hair thinning, days }\end{array}$ & \multicolumn{2}{|c|}{170} & \multicolumn{2}{|r|}{62} & \multicolumn{2}{|c|}{112} & \multicolumn{2}{|r|}{83} \\
\hline Time from onset to follow-up visit, days & \multicolumn{2}{|c|}{303} & \multicolumn{2}{|c|}{207} & \multicolumn{2}{|c|}{244} & \multicolumn{2}{|c|}{244} \\
\hline Severity Assessment & Onset & Follow-up & Onset & Follow-up & Onset & Follow-up & Onset & Follow-up \\
\hline Patient & $3 / 10$ & $\begin{array}{c}1 / 10 \\
\text { complete/ } \\
\text { near-complete } \\
\text { resolution }\end{array}$ & $3 / 10$ & $\begin{array}{l}\text { 3/10; markedly } \\
\text { improved }\end{array}$ & $5 / 10$ & $\begin{array}{l}\text { 3/10; markedly } \\
\text { improved }\end{array}$ & $5 / 10$ & $\begin{array}{c}5 / 10 ; \\
\text { completel } \\
\text { near-complete } \\
\text { resolution }\end{array}$ \\
\hline Healthcare professional & Mild & Mild & Mild & Mild & Mild & Mild & Mild & Moderate \\
\hline Location of hair thinning reported by patient & \multicolumn{2}{|c|}{ Top } & \multicolumn{2}{|c|}{$\begin{array}{l}\text { Temporal (left and right), } \\
\text { frontal (left, center, and right), } \\
\text { top, back }\end{array}$} & \multicolumn{2}{|c|}{ Top } & \multicolumn{2}{|c|}{$\begin{array}{l}\text { Temporal (left and right), } \\
\text { frontal (left, center, and right) } \\
\text { top, back }\end{array}$} \\
\hline \multirow[t]{3}{*}{ Teriflunomide continued? (Yes/No) } & \multicolumn{2}{|c|}{ Yes } & \multicolumn{2}{|c|}{ Yes } & \multicolumn{2}{|c|}{ Yes } & \multicolumn{2}{|c|}{ Yes } \\
\hline & \multicolumn{2}{|c|}{ Patient 5} & \multicolumn{2}{|c|}{ Patient 6} & \multicolumn{2}{|c|}{ Patient 7} & \multicolumn{2}{|c|}{ Patient 8} \\
\hline & Onset & Follow-up & Onset & Follow-up & Onset & Follow-up & Onset & Follow-up \\
\hline Age, years & \multicolumn{2}{|r|}{45} & \multicolumn{2}{|r|}{46} & \multicolumn{2}{|c|}{42} & \multicolumn{2}{|c|}{46} \\
\hline $\begin{array}{l}\text { Time from first dose of teriflunomide } \\
\text { to onset of hair thinning, days }\end{array}$ & \multicolumn{2}{|r|}{30} & \multicolumn{2}{|c|}{109} & & 56 & & 354 \\
\hline Time from onset to follow-up visit, days & & 338 & & 258 & & 158 & & 247 \\
\hline Severity Assessment & Onset & Follow-up & Onset & Follow-up & Onset & Follow-up & Onset & Follow-up \\
\hline Patient & $7 / 10$ & $\begin{array}{c}1 / 10 ; \\
\text { complete/ } \\
\text { near-complete } \\
\text { resolution }\end{array}$ & $7 / 10$ & $\begin{array}{l}2 / 10 ; \text { markedly } \\
\text { improved }\end{array}$ & $7 / 10$ & $\begin{array}{l}\text { 4/10; } \\
\text { somewhat } \\
\text { improved }\end{array}$ & $7 / 10$ & \begin{tabular}{|c|}
$8 / 10 ;$ \\
completel \\
near-complete \\
resolution
\end{tabular} \\
\hline Healthcare professional & Moderate & Mild & Moderate & Mild & Moderate & Mild & Moderate & Moderate \\
\hline Location of hair thinning reported by patient & $\begin{array}{l}\text { Temporal } \\
\text { (left side }\end{array}$ & $\begin{array}{l}\text { left, top, other } \\
\text { behind ear) }\end{array}$ & Not & provided & Temporal & (left and right) & & II over \\
\hline Teriflunomide continued? (Yes/No) & No, discont & nued due to rash & & Yes & & Yes & & Yes \\
\hline
\end{tabular}

Fig. 1 Examples of hair thinning at onset and follow-up. HCP healthcare professional

Over the course of follow-up, two patients discontinued teriflunomide treatment, one temporarily because of hair thinning. Three other patients discontinued treatment for other reasons: one discontinued temporarily owing to gastrointestinal upset, and the two remaining patients discontinued permanently because of rash and gastrointestinal upset.

The extent of hair loss experienced by patients treated with teriflunomide varied from mild, diffuse thinning, to loss of large clumps of hair. One patient lost her eyebrows, which did not recover, although the rest of her hair grew back.

\section{DISCUSSION}

In this observational project, hair thinning events in patients receiving teriflunomide were 


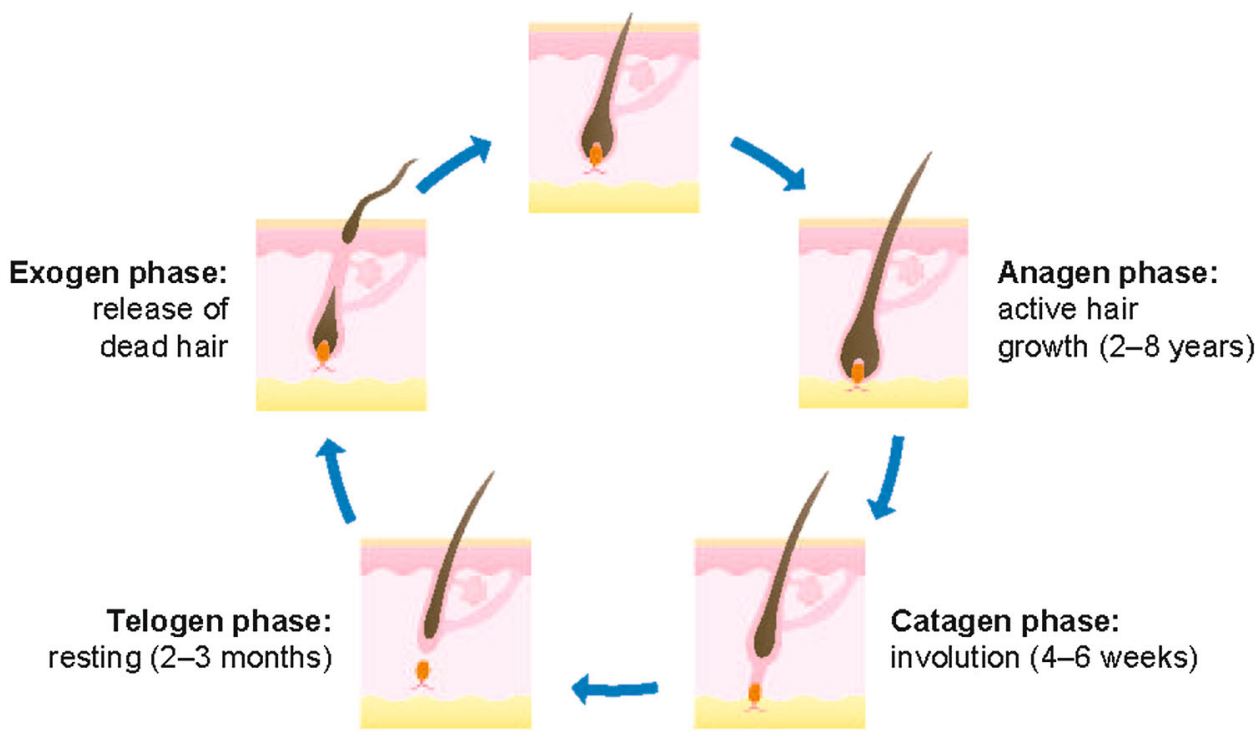

Fig. 2 Hair follicle cycle. Adapted from Harrison and Bergfeld. Diffuse hair loss: its triggers and management. Cleveland Clin J Med. 2009;76:361-7

typically mild, occurred within 3 months, and usually resolved without treatment discontinuation. These observations are consistent with those from the clinical trial program. In the 2-year TEMSO core study (NCT00134563), hair loss was reported in $11.7 \%$ of patients treated with teriflunomide, compared with $3.3 \%$ of patients receiving placebo [10]. However, in the long-term extension (NCT00803049), in which all patients were re-randomized to teriflunomide, the number of patients reporting firstonset of hair thinning was $3.6 \%$ over the first 24 weeks of the extension, and $<1 \%$ over the following 6 years [12]. Similarly, in the realworld Teri-PRO study (NCT01895335), incidence of hair thinning onset decreased from $\sim 6 \%$ of patients in the first 4 weeks of teriflunomide treatment, to $2.9 \%$ at 12 weeks and $<1 \%$ after 24 weeks [13].

In clinical trials, alopecia was reported in a small proportion of patients treated with interferon-beta [4]. The frequency of alopecia reported in patients treated with intramuscular interferon-beta in clinical trials was $4 \%$, compared with $2 \%$ of patients receiving placebo [14]. Similarly, patients receiving subcutaneous interferon-beta experienced alopecia more frequently than placebo-treated patients, although the difference was less than $2 \%$ [15].
Teriflunomide-associated hair thinning reported in this project was likely due to telogen effluvium, a common type of reversible hair loss caused by premature transition of active follicles to the resting phase, resulting in hairs being shed $\sim 3$ months later (Fig. 2). This is distinct from anagen hair loss, which is due to premature termination of anagen hair growth and is most often associated with chemotherapy or radiation therapy [3]. Medication is one of the common triggers for telogen effluvium, which is usually self-limited and does not require treatment [16].

In the opinion of one of the authors (K.R.E.), patients who experienced hair thinning had varying reactions. Since most were informed of the possibility prior to treatment initiation, there was neither distress nor upset. Some patients did express concern when hair loss began (i.e. hair coming off in a routine shower), although they were often reassured with the knowledge that hair loss was a transient effect. Patient-perceived severity of hair thinning at both onset and follow-up visits was generally more severe than assessments made by the treating HCP.

A limitation of this analysis was that hair photos were only taken when hair loss was reported and at follow-up, but were not taken 
prior to starting teriflunomide to provide a baseline.

\section{CONCLUSION}

The assessment and characterization of teriflunomide-associated hair thinning, along with patient education, could help to set appropriate expectations for patients before initiating treatment with teriflunomide.

\section{ACKNOWLEDGEMENTS}

The authors would like to thank the participants of the study and to acknowledge contributions from the following US study centers: South Tampa MS Center, Tampa, FL; Baptist Neurology, Jacksonville, FL; Rockwood Neurology Center, Spokane, WA; Neurology Center of New England, Foxboro, MA; Alabama Neurology Associates, Birmingham, AL.

Funding. This project was funded by Sanofi. Open access and article processing charges were funded by Sanofi.

Medical Writing and/or Editorial Assistance. The article was reviewed by Larisa Miller, PharmD, Alex Lublin, PhD, and Darren P. Baker, $\mathrm{PhD}$, of Sanofi. Editorial support was provided by Catherine Simonson of Fishawack Communications Ltd., and was funded by Sanofi.

Authorship. All named authors meet the International Committee of Medical Journal Editors (ICMJE) criteria for authorship of this article, take responsibility for the integrity of the work as a whole, and have given their approval for this version to be published. All authors had full access to all of the data in this study and take complete responsibility for the integrity and accuracy of the data.

Disclosures. Dr. Hendin Travis: consulting fees (Acorda, Biogen, EMD Serono, Genzyme, Novartis, Pfizer, Mallinckrodt); research support (Biogen, EMD Serono, Genzyme). Dr. Okai: consulting fees (Bayer, Biogen, EMD Serono,
Genzyme, Questcor, Teva Neuroscience); research support (Alexion, Genzyme, Novartis, Sun Pharma). Dr. Cavalier: employee of Sanofi, with ownership interest. Dr. Stam: employee of Sanofi, with ownership interest.

Compliance with Ethics Guidelines. Informed consent was obtained from all individual participants included in the study. Additional written informed consent was obtained from participants for release of their images.

Data Availability. Data included in this article have been previously presented at ECTRIMS 2015 and CMSC 2015.

Open Access. This article is distributed under the terms of the Creative Commons Attribution-NonCommercial 4.0 International License (http://creativecommons.org/licenses/ by-nc/4.0/), which permits any noncommercial use, distribution, and reproduction in any medium, provided you give appropriate credit to the original author(s) and the source, provide a link to the Creative Commons license, and indicate if changes were made.

\section{REFERENCES}

1. Brough KR, Torgerson RR. Hormonal therapy in female pattern hair loss. Int J Womens Dermatol. 2017;3:53-7.

2. Saed S, Ibrahim O, Bergfeld WF. Hair camouflage: a comprehensive review. Int J Womens Dermatol. 2017;3:S75-80.

3. Harrison S, Bergfeld W. Diffuse hair loss: its triggers and management. Cleve Clin J Med. 2009;76: $361-7$.

4. Walther EU, Hohlfeld R. Multiple sclerosis: side effects of interferon beta therapy and their management. Neurology. 1999;53:1622-7.

5. Losavio FA, Lucchini M, De Fino C, Mirabella M, Nociti V. Transient hair loss during treatment with dimethyl-fumarate for multiple sclerosis. Mult Scler Relat Disord. 2016;7:68-9. 
6. Oh J, Saidha S, Cortese I, et al. Daclizumab-induced adverse events in multiple organ systems in multiple sclerosis. Neurology. 2014;82:984-8.

7. Pacheco MF, Jacobe H, Eagar TN, Stuve O. Reversible alopecia associated with glatiramer acetate. Arch Neurol. 2010;67:1154.

8. Zimmermann JS, Buhl T, Müller M. Alopecia universalis following alemtuzumab treatment in multiple sclerosis: a barely recognized manifestation of secondary autoimmunity-report of a case and review of the literature. Front Neurol. 2017;8:569.

9. Comi G, Freedman MS, Kappos L, et al. Pooled safety and tolerability data from four placebo-controlled teriflunomide studies and extensions. Mult Scler Relat Disord. 2016;5:97-104.

10. Drug Induced Hair Loss [American Hair Loss Association website]. Available at: http://www. americanhairloss.org/drug_induced_hair_loss/. Accessed May 4, 2018.

11. O'Connor P, Wolinsky JS, Confavreux C, et al. Randomized trial of oral teriflunomide for relapsing multiple sclerosis. N Engl J Med. 2011;365: 1293-303.

12. Freedman MS, Miller AE, Comi G, et al. Outcomes of the TEMSO extension study of teriflunomide: 10.5 years of clinical results. Mult Scler. 2016;22(S3):807.

13. Coyle PK, Khatri B, Edwards KR, et al. Teriflunomide real-world safety profile: results of the phase 4 Teri-PRO study. Mult Scler. 2016;22(S3):774.

14. Avonex (interferon-beta-1a). Prescribing information. Cambridge, MA: Biogen; 2016.

15. Betaseron (interferon beta-1b). Prescribing Information. Whippany, NJ: Bayer HealthCare Pharmaceuticals; 2016.

16. Bergfeld W, Mulinari-Brenner F. Hair disorders [Cleveland Clinic Center for Continuing Education website]. Available at: https://www.cleveland clinicmeded.com/medicalpubs/diseasemanagement/ dermatology/hair-disorders/. Accessed May 4, 2018. 